\title{
Effects of noise similarity and redundancy on the information processed
} from brief visual displays ${ }^{1}$

\author{
CURTIS MCINTYRE, ROBERT FOX, ${ }^{2}$ AND JOHN NEALE ${ }^{3}$ \\ VANDERBILT UNIVERSITY
}

\begin{abstract}
When a target letter form is presented tachistoscopically and embedded in a matrix of irrelevant letter forms (noise), correct detections of the target at a given matrix size can be used to estimate indirectly the amount of information processed from the display, according to a model developed by Estes. In three experiments, the physical similarity between noise and target letters and noise-letter redundancy were varied to determine if the detection mechanism was responsive to these variables. It was found that increases in the physical differences between noise and target letters and increases in redundancy facilitated detection. It is argued that these data support a detection model that assumes parallel rather than serial information processing.
\end{abstract}

The amount of information a $S$ can extract from a briefly exposed visual display is a quantity of considerable relevance to theories of attention. Early investigations of this question were termed experiments on the span of apprehension, which was generally defined as the number of elements a $\mathrm{S}$ could simultaneously attend (Woodworth, 1938). A general conclusion derived from many studies was that the upper limit of the span was approximately 2.5 bits, even though the amount of information in the display was considerably greater.

But these earlier investigations confounded the information initially available to the $S$ with his subsequent ability to remember and report the number of elements present in a display. In order to obtain an uncontaminated estimate of the span of apprehension, Sperling (1960) devised a partial-report procedure that allowed a more adequate estimate of the span. He found that the span was an increasing monotonic function of the amount of information in the display. The conceptual model developed from this result involves two fundamental assumptions: First, all of the information in the display is available to the $S$ for several hundred milliseconds after physical termination of exposure in the form of a rapidly decaying afterimage of the initial exposure. Second, a central processing device operates upon this image to retain information before it decays. Information elements in the display are retained permanently if they are processed by the device; elements not processed prior to their decay are lost.

Working within the same conceptual framework, Estes (1965) and Estes \& Taylor (1966) developed a model of the span of apprehension that provides an indirect measure of the span and eliminates the restrictions imposed by memory and method of report. The basic measurement scheme involves a forced-choice letter recognition task, whereby one of two equiprobable letters, designated as target letters, are exposed tachistoscopically on each trial. The target letters are presented at varying positions within an array of randomly selected letters different from one another and from the target letter. Since recognition of the target letter is impeded by the array of other letters, these letters are regarded as noise. The general argument is that in order for the $S$ to make above-chance recognitions he must search the noise letters until the target letter is located. The span of apprehension, then, can be estimated from the relationship between the proportion of correct recognitions and the total number of letters in the array. The model formally asserts that the number of letters searched, i.e., the span of apprehension, is a positive monotonic function of the number of letters comprising the array. This prediction has been confirmed, as have several subsidiary predictions derived from the model.

With regard to the search mechanism that processes the letters, at least two alternative assumptions have been entertained. On the serial-processing assumption, each letter is scanned successively until the target letter is found or until some factor terminates the search. On the sampling-serial processing assumption, a sample of letters is drawn and searched exhaustively; correct recognitions can occur only when the target letter is contained in the initial sample. Certain sets of data offer support for both of these assumptions. The tests of these assumptions have served to reveal something of the general character of the search mechanism, but more specific properties are yet to be identified.

The specific property under investigation in the present series of experiments involves the effect of the character of the noise elements upon the efficiency of the search process. In previous work, the noise elements were randomly selected letters, each differing from one another, thereby forcing the search mechanism to devote an approximately equal amount of analytic power to each letter in order to decide whether it was signal or noise. But if the noise letters are all the same (redundant), or if they are physically quite dissimilar from the target letters, then these properties of redundancy and dissimilarity might be utilized by the search process to increase the efficiency of the search. Such an increase in efficiency would manifest itself empirically as an increase in thproportion of correct detections. Within the context of the Estes model of the span of apprehension, the increase in correct detections for a constant display size would be interpreted as an increase in the number of elements scanned, i.e., an increase in the span of apprehension.

One reason for believing that the search process might capitalize upon systematic differences between the noise letters and the target letter is that such differences are utilized during molar visual search involving large eye movements and long periods of searching (Neisser, 1967). These data seem to force the conclusion that a hierarchy of filters, operating in parallel, serves to protect the decision center from a substantial proportion of noise input. Clearly, a similar kind of system filter would have to be incorporated into a model of the search process for the span of apprehension if, in fact, the search process $^{4}$ proved to be sensitive to the redundancy and similarity properties of the noise letters.

Three experiments were performed to determine if the span of apprehension or the underlying search process could be influenced by manipulating the similarity and redundancy of the noise letters. In Experiment 1, there was a single noise condition, which maximized both redundancy and similarity. In 
Experiment 2, there were four noise conditions, which varied physical similarity while holding redundancy constant. In Experiment 3, there were four noise conditions, which combined levels of redundancy and of similarity. In all three experiments, the effects of redundancy and similarity were evaluated by comparison with a baseline noise condition where the noise elements differed from each other, i.e., randomly drawn letters.

\section{EXPERIMENT 1}

In this experiment, the target letters were $T$ and $F$, and the letters for the baseline noise condition were selected randomly. For the experimental noise condition only the letter $O$ was used.

\section{Method}

Subjects. The Ss were 20 introductory psychology students (12 males) with normal or corrected-normal acuity. Ss participated to fulfill a course requirement and were naive with respect to the purpose of the experiment.

Apparatus. A three-field tachistoscope (Scientific Prototype, Model GA) was employed. One field was always on and contained a fixation target composed of four 17-min black dots arranged to form a $2.5 \times 3 \mathrm{deg}$ rectangle. The dots were centered on a white background. A second field exposed the stimulus array for $90 \mathrm{msec}$. Luminance of the fixation field was $8 \mathrm{ft}-\mathrm{L}$, and the stimulus field luminance was $58 \mathrm{ft}-\mathrm{L}$, as measured by a Pritchard spot photometer.

The stimuli consisted of arrays of English letters arranged to form a hypothetical matrix of 16 spaces, each space potentially filled by one letter. The arrays differed in the number, location, and type of letters used to fill the matrix. Each matrix contained one of two target letters (either $T$ or $F$ ) and one of three amounts $(7,11$, or 13$)$ of noise letters. Each target letter was used twice in each of the positions of the potential matrix except for the restriction that the target letter could not occur in any of the four corner positions. For the baseline noise condition, the remaining matrix positions were randomly filled by 7,11 , or 13 noise letters drawn randomly from the alphabet and a complementary number of blank spaces. For the " $O$ " noise letter condition, the stimulus cards were identical in all ways to those of the baseline condition except that all noise letters were replaced by the character $O$. Thus, the original set of stimuli consisted of 288 cards (12 target positions by two target letters by two duplications by three levels of noise by two conditions of noise).
All arrays were made from capital letters typed with a Smith Corona Classic typewriter, using standard pica letters. Each letter subtended a visual angle of $18 \times 12 \mathrm{~min}$, with 18 -min horizontal and 30-min vertical spacing between characters. The letters composing each array were typed on white file cards that were mounted separately on cardboard holders that could easily be inserted into the tachistoscope. A complete matrix (four rows and four columns) covered a $2 \times 3$ deg foveal area.

Design and procedure. Each $S$ received a total of 60 practice trials in six 10-trial blocks. Each block contained five $T$ and five $F$ cards drawn randomly from one of the six matrix-size by noise-condition sets. The $S$ initiated every trial, after a verbal ready signal from $E$, by depressing a telegraph key. After a 500-msec delay, the stimulus field was displayed. The $S$ then reported which of the two critical elements he observed. At the end of the practice trials, $\mathrm{S}$ was given a 5 -min rest.

During the experiment proper, each $S$ received a total of 192 trials, consisting of two 96-trial replications. Within each replication, $S$ viewed six 16-trial blocks, two of each of the three matrix sizes; for each matrix size, one block contained baseline noise while the other contained $O$ noise. The order of occurrence of the two different noise conditions was counterbalanced over the two replications. The matrix size associated with each pair of trial blocks was selected randomly with an equal-occurrence restriction. Individual stimulus cards were randomly selected from each set for inclusion in the trial blocks.

At the end of each 32 trials, $S$ was given a 2-min rest, while the first 96 trials were followed by a $5 \cdot \mathrm{min}$ rest. An intertrial interval of approximately $15 \mathrm{sec}$ was maintained. Ss viewed the displays binocularly with natural pupis. A head-and-chin rest was used to restrict head movement.

\section{Results and Discussion}

The basic data are the mean proportions of correct recognitions combined over Ss for each of the three matrix sizes and each

\section{Table 1}

\section{Proportion of Correct Recognitions as a Function of Matrix Size and Noise Condition for Experiment 1}

\begin{tabular}{lccc}
\hline & \multicolumn{3}{c}{ Matrix Size } \\
\cline { 2 - 4 } $\begin{array}{l}\text { Noise } \\
\text { Condition }\end{array}$ & 8 & 12 & 14 \\
\hline Random & .664 & .623 & .600 \\
Redundant & .823 & .780 & .784 \\
\hline
\end{tabular}

of the two noise conditions. These data are presented in Table 1. An analysis of variance of these data reveals significant main effects for Ss $(F=7.38, \mathrm{df}=19 / 19$, $p<.001)$, matrix size $(F=6.46, \mathrm{df}=2 / 38$, $\mathrm{p}<.004)$ and noise condition $(F=98.66$, $\mathrm{df}=1 / 19, \mathrm{p}<.001)$. None of the relevant interaction terms approached significance, nor did the replication measure.

The effect of Ss simply reflects the large range of individual differences in recognition thresholds typically found in this kind of experiment. The significant effect of matrix size indicates that probability of correct recognition decreases as matrix size increases. This relationship is predicted by the Estes model, and it has been obtained in previous experiments. Since the Noise by Size interaction is not significant $(F<1)$, that relationship must operate for both noise conditions. The significant noise effect is due to the substantial increase in correct recognitions present under the $\mathrm{O}$-noise condition.

According to the Estes model, the increase in correct recognition means that a larger number of letters were processed under the $\mathrm{O}$-noise condition, i.e., the span of apprehension increased. An estimate of the span can be obtained from the proportion of correct recognitions $(\mathrm{Pc})$ at a given matrix size $(M)$. Specifically,

$$
\mathrm{Pc}_{\mathrm{c}}=\frac{\mathrm{L}}{\mathrm{M}}+\frac{1}{2}\left(1-\frac{\mathrm{L}}{\mathrm{M}}\right)
$$

where $M$ denotes matrix size and $L$ the number of letters processed. Then, by arrangement,

$$
\mathbf{L}=\mathbf{M}(2 \mathbf{P c}-1) .
$$

Under the baseline noise condition, the number of letters processed for $M=8$, $M=12$, and $M=14$, respectively, were $2.56,2.88$, and 2.80 .5 Corresponding values under the $O$-letter noise condition are $5.12,6.72$, and 7.98 .

It is clear that the $O$-noise condition leads to a marked increase in the span. This increase must be attributed to a corollary increase in the efficiency of the search process made possible by the redundancy and dissimilarity of the $\mathrm{O}$-noise letters. The obvious conclusion suggested by these data is that the search process is sensitive to global or common features of the noise background.

\section{EXPERIMENT 2}

The increase in the span of apprehension observed in Experiment 1 for the O-noise condition could be due to the redundancy of the $\mathrm{O}$-noise letter, to the physical 
differences between $O$ and the target letters, $T$ and $F$, or to some joint function of both redundancy and ph;sical similarity. Experiment 2 was designed to investigate the contribution of physical similarity between noise letters and target letters when redundancy was held constant at its maximum value, i.e., all noise letters were the same. To manipulate physical similarity, four noise letters were selected that varied in their similarity to the target letters, $T$ and $F$.

The similarity dimension was defined in terms of the number of physical features held in common between noise and target letters. Physical features refer to the classification of letters and their discriminability described by Gibson (1965). These features consist of such variables as the number and angle of straight line segments, the presence and amount of curvature, etc. An estimate of the physical similarity between any pair of letters can be made by conceptually superimposing the pair and counting the number of physical features they have in common. By this procedure, the letters $\mathrm{O}$ and $U$ were selected as being quite dissimilar to the target letters, $T$ and $F$, with $O$ being ranked as somewhat more dissimilar than $U$. The letters $I$ and $E$ were selected as being quite similar to the target letters, with I ranked as more dissimilar than E. Thus, on an ordinal scale, the common physical-feature similarity of the noise letters were ordered as $\mathrm{O}<\mathrm{U}<\mathrm{I}<\mathrm{E}$. These four letters were used to form the four experimental conditions, while a random selection of all alphabet letters formed the baseline noise condition. For all five conditions, two matrix sizes were employed: 7 noise letters plus a target and 13 noise letters plus a target.

\section{Method}

Subjects. The Ss were 20 naive paid volunteers drawn from introductory psychology courses and screened for normal binocular acuity (corrected or uncorrected).

Apparatus. The apparatus was the same as had been used in Experiment 1 . Stimuli were exposed for $90 \mathrm{msec}$ in a field with luminance of $32 \mathrm{ft}-\mathrm{L}$. The luminance of the constantly illuminated fixation field was $4 \mathrm{ft} \cdot \mathrm{L}$.

Stimulus construction for Experiment 2 adhered to the rules described for Experiment 1. Two levels (7 and 13) of noise elements were used with five noise-letter-condition displays at each level (four redundant and one random). The sets of redundant stimuli were constructed identically in all ways to both random conditions except that all noise letters were replaced by one character $(E, I, O$, or $U)$. Thus, the stimuli consisted of 240 cards (12 target positions by 2 target letters by 2 levels of noise by 5 noise-letter types).

Design and procedure. Each $S$ received 60 practice trials composed of three $T$ and three $F$ cards drawn randomly from 1 of the 10 matrix-size by noise-letter condition sets. The $S$ rested for $5 \mathrm{~min}$ after the practice trials and then began the experimental trials. Each S viewed 200 experimental trials consisting of two 100-trial replications. Each replication contained 10 randomly ordered 10-trial blocks, each block drawn from 1 of the 10 matrix-size by noise-letter condition sets. Individual stimulus cards were selected randomly for the trial blocks, with the restriction that stimulus cards viewed during the first replication did not appear in the second. Each 10-trial block was followed by a $30-\mathrm{sec}$ rest, while a 2 -min rest was allowed after every fourth 10-trial block. Both the trial procedure and the S's task were exactly the same as in Experiment 1.

\section{Results and Discussion}

The basic data are the mean proportions of correct recognitions combined over Ss for each of the two matrix sizes and each of the five noise-letter conditions. Table 2 contains these data. Analysis of variance reveals significant main effects for $S s$ $(\mathrm{F}=8.84, \mathrm{df}=19 / 19, \mathrm{p}<.001)$, matrix size $(F=12.74, d f=1 / 19, p<.002)$, and noise condition $(F=29.82, \quad d f=4 / 76$, $p<.001)$. No significant effects were obtained for replications or any of the interaction terms.

The significant Ss effect reflects the individual differences in recognition thresholds usually obtained in this kind of experiment. The significant matrix size effect reflects the expected decline in frequency of correct recognitions as a function of increasing display size.

Since a significant Matrix Size by Noise-type-condition interaction was not observed, Newman-Keuls multiple comparison tests $(p=.05)$ were applied to the significant noise condition effect. These tests indicate that the $\mathrm{O}, \mathrm{U}$, and I letter conditions differed significantly from

Table 2

Proportion of Correct Recognitions as a Function of Matrix Size and Noise Condition for Experiment 2

\begin{tabular}{llll}
\hline & & \multicolumn{2}{c}{ Matrix Size } \\
\cline { 3 - 4 } $\begin{array}{l}\text { Noise } \\
\text { Condition }\end{array}$ & & 8 & 14 \\
\hline Random & & .678 & .623 \\
Redundant & $\mathrm{O}$ & .825 & .830 \\
& $\mathrm{U}$ & .813 & .743 \\
& $\mathrm{I}$ & .773 & .690 \\
& $\mathrm{E}$ & .645 & .663 \\
\hline
\end{tabular}

one another and from the $E$ letter and the baseline noise conditions, regardless of matrix size.

As can be seen from Table 2, the similarity of the noise letters plays a substantial role in determining the proportion of correct recognitions and, in turn, the span of apprehension. The span of apprehension obtained under all conditions, as estimated by the proportion of correct recognitions, is given in Table 3. Note that the proportion of correct recognitions for the letter $O$ relative to the baseline condition is similar in magnitude to that found in Experiment 1. After $O$, the letters $U, I$, and $\mathbf{E}$ ranked themselves in terms of concommitant reductions in the proportion of correct recognitions. In fact, for the $E$ letter condition, there is no improvement in proportion of correct recognitions relative to the baseline noise-letter condition. It appears that our selection of the four letters in terms of physical similarity covers the range of similarity that might reasonably be employed with respect to letter characters. It is of some interest to note that an index of similarity could be computed for each letter in the redundant noise-letter condition by taking the difference between the proportion of correct recognitions for that letter condition and the proportion of correct recognitions found under the baseline random noise-letter condition. Such an analysis would yield results in general agreement with Gibson's scaling of physical similarity.

These data imply that the search process can utilize the physical differences and similarities between noise and target in a manner fully consistent with that assumed to operate in molar visual search. In molar visual search, it is assumed that the series of tests are applied to incoming data on the basis of physical similarity to the target element in such a way that quite dissimilar elements are rejected early in the test hierarchy, while more similar elements are rejected at later stages. The data of both Experiment 1 and Experiment 2 suggest that a filtering operation is operative during the search process similar to that assumed to occur during brief tachistoscopic exposure.

Table 3

Number of Elements Processed as a Function of Matrix Size and Noise Condition for Experiment 2

\begin{tabular}{llcc}
\hline \multirow{2}{*}{$\begin{array}{c}\text { Noise } \\
\text { Condition }\end{array}$} & \multicolumn{2}{c}{ Matrix 'Size } \\
\cline { 3 - 4 } Random & & 8 & 14 \\
\hline Redundant & $\mathrm{O}$ & 2.848 & 3.444 \\
& $\mathrm{U}$ & 5.200 & 9.240 \\
& $\mathrm{I}$ & 5.008 & 6.804 \\
& $\mathrm{E}$ & 4.368 & 5.320 \\
& 2.320 & 4.564 \\
\hline
\end{tabular}




\section{EXPERIMENT 3}

The results of Experiment 2 clearly indicate that noise and target-letter similarity under conditions of maximum noise-letter redundancy exerts a substantial influence upon the span of apprehension. In Experiment 3, the relationship between noise-letter similarity and varying levels of redundancy was investigated. To manipulate redundancy, noise letters whose similarity values were empirically determined in Experiment 2 were combined in various ways so that more than one noise letter was present in a matrix for a given condition. Specifically, two levels of redundancy were employed along with a random-letter baseline noise condition. One redundancy level involved two different noise letters, hereby designated Redundancy Level 2; the second redundancy noise-letter condition involved three different noise letters, called Redundancy Level 3. Altogether, a total of five noise-letter conditions were employed: three Level 2 redundancy conditions consisting of pairs of letters (I-O, I-U, and $\mathrm{O}-\mathrm{U})$, a Level 3 redundancy condition (I-O.U), and a random-letter baseline noise condition. For all five conditions, two matrix sizes were employed: 7 noise letters plus a target letter and 13 noise letters plus a target letter.

\section{Method}

Subjects. All of the Ss used in Experiment 2 also served in Experiment 3. To insure their availability for this experiment, they were signed up for it when they appeared for Experiment 2.

Apporatus. The apparatus, duration of stimulus exposure, and field luminances were the same as those used in Experiment 2.

Stimulus construction adhered to the rules stated previously. Five types of noise-condition displays (one baseline and four redundant) were used with two matrix sizes ( 7 and 13), producing 10 matrix-size by noise conditions. The baseline noise display set was the same as that used in Experiment 2. Three sets of stimuli (I-O, $I-U$, and $0 . U$ ) were constructed at both matrix-size levels by replacing the positions heid by the noise elements in the random stimuli with one or the other of two characters. For example, a stimulus display for the : O-U letter pair at Matrix Size 7 would consist of one signal element ( $T$ or F) and either three Os and four Us or four Os and three Us. The Redundancy Level 3 condition ( $\mathrm{l}-\mathrm{O}-\mathrm{U})$ at each matrix size was obtained by replacing the noise positions within the matrices with selections from a pool of three characters instead of two. Assignment of the characters to the various positjons within the matrix was random, with the restriction that the frequency of occurrence of the noise letters was balanced within the set of displays.

Design and procedure. The design and procedure were exactly the same as those used in Experiment 2.

\section{Results and Discussion}

The basic data are the mean proportions of correct recognitions combined over Ss for each of the two matrix sizes and each of the five noise conditions. Table 4 contains these data. Analysis of variance reveals significant main effects for Ss $(\mathrm{F}=9.42$, df $=19 / 19, \mathrm{p}<.001)$, matrix size $(F=18.14, \mathrm{df}=1 / 19, \mathrm{p}<.001)$, and noise condition $(F=4.52$, $\mathrm{df}=4 / 76$, $\mathrm{p}<.003)$. No significant effects were obtained for replications or for any of the interactions.

The significant Ss effect reflects individual differences in recognition thresholds, and the significant matrix-size effect indicates the predictable decline in correct recognitions as the display size increases.

By the Newman-Keuls multiple comparison test $(p=.05)$, only the O.U letter condition differs significantly from the other conditions. The I-O, I-U, and I-O-U conditions did not differ significantly from the random-letter baseline condition. Comparison of the proportion of correct responses for the I, $O$, and $U$ conditions of Experiment 2 (see Table 2) with a comparable letter condition of Experiment 3 (see Table 4) reveals a general reduction in recognition accuracy for Experiment 3 . Since in both experiments the Ss, apparatus, and related variables were identical, this reduction is most likely attributable to the redundancy levels of the noise letters employed in Experiment 3.

One way to assess the role of redundancy in determining the performance obtained in Experiment 3 is to compute the proportion of correct responses expected on the basis of physical similarity alone. An index of similarity can be secured from the data of Experiment 2 by comparing the performance for each of the letters $I, O$, and $U$ with performance obtained under the random-letter baseline

Table 4

Proportion of Correct Recognitions as a Function of Matrix Size and Noice Condition for Experiment 3

\begin{tabular}{llcc}
\hline & \multicolumn{2}{c}{ Matrix } & Size \\
Noise & & 8 & 14 \\
\hline Condition & & .680 & .668 \\
Random & & .705 & .668 \\
Redundant & $1-0$ & .760 & .643 \\
& $1-U$ & .773 & .740 \\
& $0-U$ & .735 & .665 \\
\hline
\end{tabular}

rdition. In other words, the difference in c rect recognitions between the baseline condition and the redundant condition is d. termined for each letter and each matrix size; the similarity index for a particular letter is simply that difference. To illustrate, recall that for Matrix Size 8 (in Experiment 2) the random-letter baseline condition recognition score was .678 and for the O-noise condition the Matrix Size 8 recognition score was 825 . Therefore, the difference between these scores, .147 , is the similarity index for the letter $O$. In the sid le fashion, a similarity index can be computed for all of the redundant letters employed in Experiment 2.

Given the similarity indices of the separate letters, the next step is to consider how combinations of these letters would operate in Experiment 3 to determine recognition performance. The simplest assumption is that the similarity index for any combination of letters is the mean of the similarity indices of the individual letters, so long as the letters appear equally of ten. To illustrate, the similarity index for the letter I at Matrix Size 8 is .095; the index for the letter $O$, obtained under comparable conditions, is .147 . Therefore, the similarity index for the combination I-O is the mean of these values, . 121 .

If similarity is the only factor controlling recognition performance in Experiment 3, it should be possible to predict the results by adding the similarity indices for each letter combination to the random-letter baseline condition for each matrix size. To obtain these predicted scores, the random-letter baseline conditions for both Experiments 2 and 3 were averaged to increase the reliability of the estimates of baseline performance; the mean baseline score for Matrix Size 8 was .679; for Matrix Size 14, the baseline score was .646 . The similarity scores were then added to these mean baseline estimates, yielding predicted results for Experiment 3; these predictions are given in Column 2 of Table 5.

It is clear that these predicted values consistently overpredict the obtained recognition scores by about $8 \%$. The magnitude and direction of the difference strongly suggests that the less-than-maximum tedundancy of the twoand three-fetter combinations is responsible for the difference. This assertion follows on the assumption that the presence of different noise letters in the matrix necessarily impedes the efficiency of the search process. A simple way to conceptualize the nature of the impediment is to assume that the analytic power of the search process is directly reduced by the number of different noise letters present in the matrix. Thus, for 
Table 5

Comparison of Obtained Proportions of Correct Recognitions for Experiment 3 with Those Predicted on the Baxis of Similarity Alone and on Similarity and Redundancy

\begin{tabular}{|c|c|c|c|c|c|c|}
\hline \multirow[b]{2}{*}{$\begin{array}{c}\text { Noise } \\
\text { Condition }\end{array}$} & \multirow[b]{2}{*}{$\begin{array}{c}\text { Matrix } \\
\text { Size }\end{array}$} & \multirow[b]{2}{*}{ Obtained } & \multicolumn{3}{|c|}{ Proportion Correct Responses } & \multirow[b]{2}{*}{ Difference } \\
\hline & & & $\begin{array}{l}\text { Predicted } \\
\text { Similarity }\end{array}$ & Difference & $\begin{array}{c}\text { Predicted } \\
\text { Similarity } \\
\text { and Redundancy }\end{array}$ & \\
\hline Random & $\begin{array}{r}8 \\
14\end{array}$ & $\begin{array}{l}.680 \\
.668\end{array}$ & & & & \\
\hline $\begin{array}{l}\text { Redundant } \\
\mathrm{I}-\mathrm{O}\end{array}$ & $\begin{array}{r}8 \\
14\end{array}$ & $\begin{array}{l}.705 \\
.668\end{array}$ & $\begin{array}{l}.800 \\
.783\end{array}$ & $\begin{array}{l}.095 \\
.115\end{array}$ & $\begin{array}{l}.740 \\
.714\end{array}$ & $\begin{array}{l}.035 \\
.046\end{array}$ \\
\hline $\mathbf{I}-\mathbf{U}$ & $\begin{array}{r}8 \\
14\end{array}$ & $\begin{array}{l}.760 \\
.643\end{array}$ & $\begin{array}{l}.794 \\
.740\end{array}$ & $\begin{array}{l}.034 \\
.097\end{array}$ & $\begin{array}{l}.736 \\
.693\end{array}$ & $\begin{array}{r}-.024 \\
.050\end{array}$ \\
\hline $\mathbf{O}-\mathbf{U}$ & $\begin{array}{r}8 \\
14\end{array}$ & $\begin{array}{l}.773 \\
.740\end{array}$ & $\begin{array}{l}.820 \\
.810\end{array}$ & $\begin{array}{l}.047 \\
.070\end{array}$ & $\begin{array}{l}.750 \\
.728\end{array}$ & $\begin{array}{r}-.023 \\
-.012\end{array}$ \\
\hline $\mathbf{I}-\mathbf{O}-\mathbf{U}$ & $\begin{array}{r}8 \\
14\end{array}$ & $\begin{array}{l}.735 \\
.665\end{array}$ & $\begin{array}{l}.805 \\
.777\end{array}$ & $\begin{array}{l}.070 \\
.112\end{array}$ & $\begin{array}{l}.721 \\
.690\end{array}$ & $\begin{array}{r}-.014 \\
.025\end{array}$ \\
\hline \multicolumn{4}{|c|}{ Mean difference between obtained and predicted } & .080 & & .010 \\
\hline
\end{tabular}

two-letter combinations (Redundancy Level 2), such power is reduced to one-half that available under maximum redundancy conditions; similarly, for three-letter combinations, the power is reduced to one-third. The reduction of analytic power might be conceptualized as forcing the system to make additional tests before deciding that a target letter was present. For example, in the case of Redundancy Level 1, where only a single letter is present, say, the letter $O$, it is necessary to test only the $O$ against the target letters $T$ and F. But in the case of Redundancy Level 2 with, say, the letters $O$ and $I$ present, then both $O$ and I must be tested against the target letters. Similarly, for Redundancy Level 3 with, say, the letters $\mathbf{O}, 1$, and $U$ present, then all three of these noise letters must be tested against the target letters. ${ }^{6}$

If redundancy does act in this manner, the recognition scores predicted on the basis of similarity alone must be adjusted for the redundancy level of the letter combinations. In the case of the three two-letter combinations (I-O, I-U, and O-U), the predicted values are divided by 2 . In the same way, the predicted values for the three-letter combinations are divided by 3 . The results of this division are given in Column 4 of Table 5, and are the predicted recognition scores based upon similarity and redundancy combined. It is clear that this adjustment markedly reduces the difference between predicted performance and obtained performance. The overall difference is approximately $1 \%$, and the direction of the differences is not systematic.

Although this analysis of the contribution of redundancy is clearly post hoc, it does account reasonably well for the results of Experiment 3, especially so since the analysis ignores other variables known to influence recognition performance on this task, such as position of the target letter within the matrix and the number of noise letters surrounding the variable. The contribution of these variables has recently been described by Wolford, Wessel, and Estes (1968).

Further, the analysis suggests that both similarity and redundancy operate together to determine detection performance in a simple way. Namely, the contribution of the noise letters to detection performance is determined by the similarity index of the noise letters divided by the redundancy level of the noise letters in the matrix.

The effect of redundancy upon detection seems to suggest that all the letters in the matrix are processed simultaneously up to the point where it is possible to decide if the letters are all the same, are all different, and so on. The effect of similarity seems to suggest that the discrimination of target letters from noise letters proceeds by an analysis of classes of structural attributes or features common to all of the letters rather than by an analysis of the unique combination of attributes characteristic of a specific letter.

The hypothetical functions of both redundancy and similarity imply an information-processing system in which several analyses are concurrently made upon a set of inputs, a conception that seems to reflect what is meant by the idea of parallel processing of information. Conversely, the effects of redundancy and similarity do not seem consistent with a processing system that sequentially analyzes each noise letter, a conception that seems to reflect what is meant by the idea of serial processing of information.

Accordingly, the general theoretical conclusion derived from the results of Experiments 1 through 3 is that some version of a parallel processing system must be incorporated into the Estes model. Wolford, Wessel, and Estes (1968) reached a similar conclusion based upon data target letter, the so-called noise-density derived from the use of a redundant target technique. Yet, as Townsend (1968) has noted, fundamental differences between parallel and serial models are subtle and difficult to discriminate empirically.

\section{REFERENCES}

ESTES, W. K. A technique for assessing variability of perceptual span. Proceedings ofthe National Academy of Sciences, 1965, 53, $2,403-407$.

ESTES, W. K., \& TAYLOR, H. A. Visual detection in relation to display size and redundancy of critical elements. Perception \& Psychophysics, 1966, 1, 9-16.

GIBSON, E. J. Learning to read. Science, 1965 , 148, 1066-1072.

NEISSER, U. Cognitive prychology. New York: Appleton-Century-Crofts, 1967.

SPERLING, G. The information available in brief visual presentations. Psychological Monographs, 1960, 74(Whole No. 498).

TOWNSEND, J. T. Mock parallel and serial models and experimental detection of these. Paper presented at the meeting of the Midwestern Mathematical Psychology Association, Bloomington, April 1968.

WOLFORD, G. L., WESSEL, D. L., \& ESTES, W. $K$. Further evidence concerning scanning and sampling assumptions of visual detection models. Perception \& Psychophysics, 1968, 3, 439-444.

WOODWORTH, R. S. Experimental psychology. New York: Holt, 1938.

\section{NOTES}

1. This research was supported in part by PHS Grant NB 07619. The authors wish to thank Joe Lappin for his helpful comments on i.. manuscript.

2. Reprints may be requested from Robert Fox, Department of Psychology, Vanderbilt University, Nashville, Tennessee 37203.

3. Address: Department of Psychology, State University of New York at Stony Brook, Stony Brook, Long Island, New York 11790.

4. Note that the term "search process," as we use it, encompasses potentially separable subcomponents of the system, such as registration, encoding, etc., and no systematic attempt is made to distinguish among them.

5. It should be noted that for the random-noise condition, the probability of correct detection is approximately $10 \%$ lower for the present study than that obtained by Estes and Taylor (1966). This reduction is probably attributable to the larger display size (5.5 $\times 5.5 \mathrm{deg}$ ) employed by them rather than to the differences in exposure duration and luminance between the studies, since differences in these variables at the levels utilized do not contribute materially to detectability.

Note also that within the Estes model, even relatively small differences in hit rate (probability of correct recognition) lead to large differences in the estimate of the number of elements processed. Thus, the $10 \%$ reduction in hit rate observed in the present study resulted in estimates of the number of elements processed that were approximately half the size of those reported by Estes and Taylor.

6. This analysis can be extended to the case where minimal redundancy occurs, i.e., noise elements are selected at random without replacement. Here, the expected result would be the hit rate obtained for the random-noise condition, since the mean of the similarity indices should approach zero.

(Accepted for publication September 10, 1969.) 